\title{
Factores sociodemográficos, conocimiento sobre inmunizaciones asociados al cumplimiento del calendario de vacunación en madres de niños menores de un año
}

\author{
Sociodemographic factors, knowledge about immunizations associated with \\ compliance with the vaccination schedule in mothers of children under one year of
} age

\author{
Noemi Quispe Chambi', Rosa Valencia Guevara²
}

\begin{abstract}
RESUMEN
Objetivo: Determinar la relación entre los factores sociodemográficos, conocimiento sobre inmunizaciones asociados al cumplimiento del calendario de vacunación en madres de niños menores de un año de un hospital nacional - Lampa, 2019. Metodología: De enfoque cuantitativo, diseño no experimental, de corte transversal, y de tipo correlacional.; la muestra estuvo conformada por 300 madres de niños menores de un año. Para la recolección de datos se aplicó un cuestionario elaborado por Izquierdo (2014) y una lista de cotejo. Resultados: Que existe una correlación significativa $(p<.05)$ entre conocimiento sobre inmunizaciones y cumplimiento del calendario de vacunación. Respecto a los factores sociodemográficos muestra que existe correlación significativa $(p<.05)$ entre el grado de instrucción y cumplimiento del calendario de vacunación, con respecto al número de hijos y cumplimiento existe una correlación significativa $(p>.05)$. Conclusión: Existe correlación significativa entre el nivel de conocimientos sobre inmunizaciones y el cumplimiento en el calendario de vacunación. Con respecto a los factores sociodemográficos grado de instrucción y el número de hijos si están asociadas al cumplimiento del calendario de vacunación.
\end{abstract}

Palabras claves: Inmunización, conocimiento, factores sociodemográficos, calendario de vacunación..

\begin{abstract}
Objective: bjective: to determine the relationship between sociodemographic factors, knowledge of immunizations associated with compliance with the vaccination schedule in mothers of children under one year of age in a national hospital - Lampa, 2019. Methodology: quantitative approach, nonexperimental, cross-sectional design, and of a correlational type. The sample consisted of 300 mothers of children under one year of age. For data collection, a questionnaire prepared by Izquierdo (2014) and a checklist were applied. Results: that there is a significant correlation $(p<.05)$ between knowledge about immunizations and compliance with the vaccination schedule. Regarding sociodemographic factors, it shows that there is a significant correlation $(p<.05)$ between the level of education and compliance with the vaccination schedule, with respect to the number of children and compliance, there is a significant correlation ( $p>.05)$. Conclusion: there is a significant correlation between the level of knowledge about immunizations and compliance with the vaccination schedule. Regarding sociodemographic factors, degree of education and the number of children if they are associated with compliance with the vaccination schedule.
\end{abstract}

Keywords: Immunization, knowledge, sociodemographic factors, vaccination schedule.

${ }^{1}$ Universidad Peruana Unión, Juliaca, Perú

Orcid ID: 0000-0002-1847-2095

${ }^{2}$ Universidad Peruana Unión, Juliaca, Perú

Orcid ID: 0000-0003-2663-9550 


\section{INTRODUCCIÓN}

Las vacunas previenen enfermedades, discapacidades y muertes por enfermedades inmunoprevenibles. La tasa mundial de cobertura de vacunación se ha estancado en el $86 \%$. El uso de vacunas nuevas o infrautilizadas está aumentando. Si se mejora la cobertura de vacunación mundial se podría prevenir 1,5 millones de muertes (Organizacion Mundial de la Salud, 2018).

La última encuesta nacional de salud materno infantil (ENSMI), así como la tercera evaluación del cumplimiento de los objetivos de desarrollo del milenio (ODM), ambas recientemente publicadas, demostraron preocupantes retrocesos en la aplicación de vacunas a niños menores de un año (Organización Panamericana de la Salud, 2015)

Existen muestras múltiples de las limitantes que se evidencia en el proceso de vacunación a nivel de la región Puno, ya que el $30 \%$ de la población aún conservan los mitos, costumbres y creencias negativas y falsas acerca de las vacunas. Analizando la cobertura de vacunación a nivel de la región se observa gran preocupación por la baja cobertura y colocación de vacunas a niños menores de un año. ya que existe gran rechazo a las vacunas convirtiéndose en una población vulnerable a desarrollar enfermedades que bien se pueden prevenir con las vacunas que existen y están disponibles en todos los establecimientos de salud, hospitales y clínicas de todo el Perú. (Direccion Regional de Puno, 2018)

El objetivo del presente trabajo de investigación es determinar la relación entre los Factores sociodemográficos, conocimiento sobre inmunizaciones asociados al cumplimiento del calendario de vacunación en madres de niños menores de un año del Hospital Antonio Barrionuevo - Lampa, 2019.

\section{METODOLOGÍA}

El presente estudio tuvo un enfoque cuantitativo, diseño no experimental, de tipo descriptivo correlacional y de corte trasversal.

\section{Participantes}

Se aplicó el muestreo no probabilística, utilizando los criterios de inclusión y exclusión. La muestra estuvo conformada por 300 madres con niños menores de un año.

\section{Instrumento}

Para la variable conocimientos y factores sociodemográficos se ha utilizado un cuestionario elaborado por Izquierdo (2014), el instrumento fue sometido a juicio de expertos para su validación. Según el coeficiente Kuder Richarson KR-20, el instrumento de conocimientos tiene una confiabilidad de 0,716.

Para la variable cumplimiento, se verificó el carnet de vacunación y se evaluó con una lista de cotejo con los términos de si cumple y no cumple. Este instrumento es válido; según el coeficiente Kuder Richarson KR-20, el instrumento de cumplimiento tiene una confiabilidad de 0,741.

\section{Procesamiento de datos}

El procesamiento y análisis de datos se realizó con el programa estadistico SPSS versión 23, los resultados fueron presentados en tablas de frecuencia. Para la inferencia de los resultados se aplicó el coeficiente de correlación de Tau C de Kendall. El análisis estadístico dado que la presente investigación es de tipo correlacional, aplicaremos el coeficiente de correlación de Tau $C$ de Kendall donde la relación de las variables toma valores comprendidos $-1 \mathrm{y}+1$ pasando por 0 .

\section{RESULTADOS}

La tabla 1 muestra que existe una correlación directa y significativa $(p<.05)$ entre conocimiento sobre inmunizaciones y cumplimiento del calendario de vacunación de las madres de niños menores de un año que acuden un hospital nacional de Lampa 
Tabla 1

Conocimiento sobre inmunizaciones y cumplimiento del calendario de vacunación de las madres de niños menores de un año que acuden a un hospital nacional, Lampa - 2019.

\begin{tabular}{|c|c|c|c|c|}
\hline & & & $\begin{array}{l}\text { Conocimiento so- } \\
\text { bre inmunizaciones }\end{array}$ & $\begin{array}{l}\text { Cumplimiento del } \\
\text { calendario de va- } \\
\text { cunación }\end{array}$ \\
\hline \multirow[t]{6}{*}{ Tau_c de Kendall } & \multirow{3}{*}{$\begin{array}{l}\text { Conocimiento sobre inmu- } \\
\text { nizaciones }\end{array}$} & Coeficiente de correlación & 1,000 &, $220\left(^{* \star}\right)$ \\
\hline & & Sig. (bilateral) & & ,000 \\
\hline & & $\mathrm{n}$ & 300 & 300 \\
\hline & \multirow{3}{*}{$\begin{array}{l}\text { Cumplimiento del calen- } \\
\text { dario de vacunación }\end{array}$} & Coeficiente de correlación &, $220\left(^{* *}\right)$ & 1,000 \\
\hline & & Sig. (bilateral) &, 000 & . \\
\hline & & $\mathrm{n}$ & 300 & 300 \\
\hline
\end{tabular}

** La correlación es significativa al nivel 0,01 (bilateral).

La tabla 2 muestra que existe una correlación directa y significativa $(p<.05)$ entre el grado de instrucción y cumplimiento del calendario de vacunación de las madres de niños menores de un año que acuden un hospital nacional de Lampa.

Tabla 2

Grado de instrucción y cumplimiento del calendario de vacunación de las madres de niños menores de un año que acuden a un hospital nacional, Lampa - 2019

\begin{tabular}{cllll} 
Tau_c de Kendall & $\begin{array}{l}\text { Cumplimiento del ca- } \\
\text { lendario de vacunación }\end{array}$ & $\begin{array}{l}\text { Coeficiente de } \\
\text { correlación } \\
\text { lendario de vacunación }\end{array}$ & 1,000 &, $131\left(^{*}\right)$ \\
& $\begin{array}{l}\text { Sig. (bilateral) } \\
\text { Grado de instrucción }\end{array}$ & $\cdot$ &, 015 \\
& $\mathrm{~N}$ & 300 & 300 \\
& $\begin{array}{l}\text { Coeficiente de } \\
\text { correlación } \\
\text { Sig. (bilateral) }\end{array}$ &, $131\left(^{*}\right)$ & 1,000 \\
& $\mathrm{~N}$ &, 015 & 300 \\
\hline
\end{tabular}

* La correlación es significativa al nivel 0,05 (bilateral).

La tabla 3 muestra que existe una correlación indirecta y significativa $(p>.05)$ entre el número de hijos que tienen la madres y cumplimiento del calendario de vacunación de las madres de niños menores de un año que acuden al Hospital Antonio Barrionuevo. 
Tabla 3

Número de hijos y cumplimiento del calendario de vacunación de las madres de niños menores de un año que acuden un hospital nacional, Lampa - 2019.

\begin{tabular}{|c|c|c|c|c|}
\hline & & & Número de hijos & $\begin{array}{l}\text { Cumplimiento del ca- } \\
\text { lendario de vacunación }\end{array}$ \\
\hline \multirow[t]{6}{*}{ Tau_c de Kendall } & Número de hijos & $\begin{array}{l}\text { Coeficiente de } \\
\text { correlación }\end{array}$ & 1,000 &,$- 259\left(^{* *}\right)$ \\
\hline & & Sig. (bilateral) & . & ,000 \\
\hline & & $\mathrm{n}$ & 300 & 300 \\
\hline & $\begin{array}{l}\text { Cumplimiento } \\
\text { del calendario de } \\
\text { vacunación }\end{array}$ & $\begin{array}{l}\text { Coeficiente de } \\
\text { correlación }\end{array}$ &,$- 259\left(^{* *}\right)$ & 1,000 \\
\hline & & Sig. (bilateral) & ,000 & . \\
\hline & & $\mathrm{n}$ & 300 & 300 \\
\hline
\end{tabular}

* La correlación es significativa al nivel 0,01 (bilateral).

\section{DISCUSIÓN}

Con respecto al conocimiento y cumplimiento del calendario de vacunación, los resultados son similares a los encontrados por Chafloque, Escobedo y Vicente (2018) en su estudio realizado en Lima, cuyos resultados evidenciaron que el $80,6 \%$ de las madres, tienen conocimiento bajo e incumplimiento del calendario de vacunación. Concluyeron en que existe relación directamente proporcional entre el conocimiento y el incumplimiento.

Así mismo la variable grado de instrucción y el cumplimiento del calendario de vacunación, los resultados son similares a los encontrados por Blas(2017) donde el nivel educativo superior de la madre fue del $80 \%$ y están ampliamente relacionados en el cumplimiento puntual de la vacunación de sus niños menores de 1 año. Por otro lado la variable del número de hijos y el cumplimiento del calendario de vacunación. Los resultados obtenidos guardan relación con los encontrados por Izquierdo (2014) al analizar los factores sociodemográficos: edad, número de hijos, procedencia, ingreso mensual, grado de instrucción y ocupación con el cumplimiento del calendario vacunal, se observa que todos tienen relación significativa.

\section{Declaración de financiamiento y de conflictos de interés:}

El estudio fue financiado por los autores, quienes declaran no tener conflictos de interés.

\section{Correspondencia}

Noemi Quispe Chambi

Correo electrónico:

noemi.upeu.1243@gmail.com

Rosa Valencia Guevara

Correo electrónico:

rosela16valencia@gmail.com

\section{REFERENCIAS BIBLIOGRÁFICAS}

Blas, C. E. (2017). Factores relacionados al cumplimiento del calendario de vacunación de madres primerizas de menores de 1 año en un Hospital Nacional de Lima 2016. (Tesis de licenciatura, Universidad Nacional Mayor de san Marcos) Recuperado de: http://cybertesis. unmsm.edu.pe/handle/cybertesis/6407

Chafloque, E., Escobedo, A. C., y Vicente, Y. M. (2018). Relación entre el conocimiento de las madres sobre las vacunas y el cumplimiento del calendario de vacunación en el lactante menor de un año en el establecimiento de Salud Materno Infantil de Zapallal, 2017. (Tesis de licenciatura, Universidad Peruana Cayetano Heredia) Recuperado de: http://repositorio.upch.edu.pe/bitstream/handle/ upch/3726/Relacion_ChafloquePuicon_Estefany. pdf? sequence $=1$ \&is $\bar{A}$ llowed $=y$

Direccion Regional de Puno. (2018). cobertura regional de inmunizacines Puno.

Izquierdo, O. R. (2014). Conocimiento den madres de niños menores de un año sobre inmunizaciones y el cumplimiento del calendario vacunal. (Tesis para licenciatura ,Universidad Ricardo Palma). 
Recuperado de http://repositorio.urp.edu. pe/bitstream/handle/urp/342/Moukhallalele_ rf.pdf? sequence=1\&isAllowed=y

Organizacion Mundial de la Salud. (2018). Covertura Vacunal.
Organización Panamericana de la Salud. (2015). Análisis de Situación: Inmunizaciones y Vacunas. Guatemala: Oficina Sanitaria Panamericana.

Recibido: 07/02/2020

Aceptado: 10/04/2020 